\title{
Vigilancia de eventos adversos a vacunas
}

\author{
Katia Abarca V.
}

\section{Vaccines: Adverse events surveillance}

The objective of the surveillance systems of vaccine adverse events is monitoring events temporally related to vaccination, to evaluate their causality with the vaccine and to detect events after the introduction of new vaccines. The ultimate goal of these systems is to provide the population with the best safety standard of the vaccines. The surveillance system can be passive (spontaneous reports) or active (active follow up of vaccinees); the majority of them are passive. The article gives a brief review of the most known vaccine adverse events surveillance systems, including the American Vaccine Adverse Events Report System (VAERS), the Canadian and European systems, a commentary about the Chilean one, highlighting its main advantages and also its limitations.

Key words: Vaccines; surveillance; adverse events.

Palabras claves: Vacunas; vigilancia; eventos adversos.
$\mathrm{E}$ 1 uso masivo de vacunas ha impactado enormemente en la salud de la población, reduciendo en forma notoria la incidencia de importantes enfermedades infecciosas, como sarampión, tétanos, difteria, poliomielitis, coqueluche, síndrome de rubéola congénita, enfermedad invasora por Haemophilus influenzae tipo b (Hib), e incluso logrando la erradicación de la viruela. Al disminuir la frecuencia de estas enfermedades en forma tan dramática, la población, y también los médicos, van perdiendo la capacidad de percibir su importancia. Así, los eventos adversos asociados a las vacunas (EAAV), si bien de baja frecuencia, comienzan a adquirir mayor importancia relativa, siendo poco tolerable para la opinión pública experimentar una noxa por una vacuna usada para prevenir una enfermedad ya casi inexistente. El riesgo de eventos adversos pasa entonces a ser un riesgo inmediato, comparado con el riesgo de padecer la enfermedad, que es muchas veces desconocido y al menos diferido en el tiempo.

La forma en que la población asimila la ocurrencia de EAAV es de la mayor importancia para evitar la pérdida de confianza en estos productos. Los ejemplos de situaciones en que la población ha perdido confianza en ciertas vacunas han demostrado que ocurre una baja en la cobertura de inmunización, con el consiguiente aumento de la enfermedad en cuestión. Una clara muestra de ello ocurrió en Inglaterra en la década de los 70, en que se asoció la vacuna DTP con daño cerebral; como consecuencia, la cobertura de vacunación cayó a valores cercanos al 30\% y se observaron grandes y graves brotes de coqueluche, que sólo fueron controlados con la recuperación de la cobertura de vacunación.

En distintos países se han desarrollado sistemas de Vigilancia de Eventos Adversos a Vacunas (VEAV) con el fin de monitorizarlos, evaluar su causalidad con la vacuna (principalmente importante en el caso de eventos adversos graves- (en inglés se emplea el término: severe adverse events o SAE), realizar vigilancia post marketing de nuevas vacunas, para detectar posibles EAAV no conocidos, y prevenir la pérdida de confianza en las vacunas que puede llevar a disminución de su cobertura y la reaparición de epidemias; en otras palabras, garantizar altos estándares de seguridad de las vacunas administradas a la población.

Es importante tener en cuenta los siguientes aspectos claves en relación a los EAAV:

- No existe la vacuna ideal que proteja $100 \%$ a todos los vacunados y sea totalmente segura en todos ellos.

- Los EAAV son generalmente leves y transitorios.

- La mayoría de los EAAV reportados tienen relación temporal, pero no son causados por la vacuna (coincidentes, errores de administración, etc).

- No es posible prever el desarrollo de un EAAV leve o grave-(SAE), las contraindicaciones sólo pueden minimizar el riesgo.
Pontificia Universidad Católica

de Chile. Santiago, Chile

Escuela de Medicina,

Departamento de Pediatría

Recibido: 18 agosto 2005

Aceptado: 4 enero 2006

Correspondencia a: Katia Abarca Villaseca katia@med.puc.cl 
La definición internacionalmente utilizada de evento adverso grave (SAE) es: todo evento capaz de producir muerte, amenazar la vida, provocar hospitalización o prolongación de una hospitalización, incapacidad persistente o anomalías congénitas o defectos de nacimiento relacionado al uso de una vacuna o medicamento.

\section{Sistemas de vigilancia de eventos adversos a vacunas (VEAV)}

Los sistemas de VEAV pueden ser:

- Vigilancia pasiva: Sistemas de reportes espontáneos

- Vigilancia activa: Seguimiento activo post vacunación

La mayoría de los sistemas existentes corresponden a sistemas de vigilancia pasiva.

En Chile, existe un sistema de VEAV implementado en 1994, en que se reportan EAAV en un formulario especialmente diseñado. Los reportes son efectuados mayoritariamente por enfermeras del Programa Ampliado de Inmunización (PAI). La recepción de la información se hace en el PAI central, desde donde en caso de tratarse de un evento grave (SAE) se establece un contacto con el médico tratante hasta su resolución. El estudio y la determinación de causalidad se realizan en el nivel local. La información recopilada por este sistema no es de acceso público.

En el resto del mundo, el sistema de VEAV más conocido es el de E.U.A., donde el reporte de ciertos EAAV es mandatorio por ley y existe compensación a las víctimas de injurias por vacunas de uso rutinario (National Vaccine Injury Compensation Program VICP, 1986). El sistema de vigilancia se conoce por las siglas VAERS (Vaccines Adverse Events Report System) y fue creado en 1990 en conjunto por las agencias a cargo de las inmunizaciones (CDC) y del control de alimentos y fármacos (FDA). Los reportes se hacen a través de un formulario especial por médicos, personal de salud, fabricantes de vacunas y también por pacientes o padres. Los eventos se han codificado en un sistema conocido como COSTART, que contiene más de 1.300 términos médicos. El formulario está disponible con correo prepagado, además existe un sistema gratuito de apoyo las 24 horas del día para obtener formularios, asistencia a llenado y resolución de dudas. Personal del CDC y de la FDA analizan periódicamente la información y realizan un seguimiento activo de los casos graves Los datos se encuentran disponibles al público ${ }^{1}$.

Muchos otros países cuentan con sistemas de VEAV: como Canadá con un sistema implementado en 1987 , de vigilancia pasiva complementada con vigilan- cia activa de hospitalizaciones por EAAV conocido como IMPACT (Immunization Monitoring Program Active). Inglaterra que emplea el sistema llamado "yellow cards", en que se reportan tanto eventos adversos relacionados a fármacos como a vacunas; ciertos fármacos y vacunas se vigilan en forma más intensiva y son reconocidas por un triángulo negro; Italia, Francia, España, Australia, entre otros.

También existen sistemas que integran la información de varios países: como el EUVAX: European Union Vaccinovigilance, que incluye a los países de la Unión Europea más Suiza y Noruega. Asimismo, la OMS ha elaborado una guía para implementar monitoreos de EAAV (WHO EPI), que persigue, predominantemente, la detección de errores programáticos y una rápida respuesta y equipos de evaluación de brotes de SAEs o muertes. En el año 2003, la OMS y UNICEF crearon una red de seguridad en vacunas, con información confiable en seguridad de vacunas y un Comité de Evaluación de Seguridad (GACVS). En 1997, la OMS estimó que sólo $12(14 \%)$ de los países con Programas de Inmunización (EPI) tienen un sistema de VEAV implementado.

\section{Experiencia con el sistema VAERS, E.U.A.}

Al año 2002 se habían recibido más de 140.000 reportes desde su inicio, período en que se administró alrededor de 1,9 billones de dosis vacunas; esto corresponde a más de 10.000 reportes por año. Las fuentes de los reportes son los manufacturadores de vacunas (42\%), personal de salud (30\%), departamentos de salud (12\%) y pacientes o padres $(7 \%)$. El $45 \%$ de los reportes corresponde a niños bajo 5 años de edad, 39\% ha recibido más de una vacuna. Doce por ciento de los reportes corresponden a SAEs.

Aportes del VAERS. Numerosos son los aportes de este sistema de VEAV:

- Provee información que reafirma el perfil de seguridad de vacunas recientemente licenciadas. Esto es relevante considerando el número limitado de sujetos vacunados en los estudios clínicos pre-licencia de vacunas. Numerosas publicaciones utilizando el VAERS han reafirmado la seguridad de vacunas como anti-varicela ${ }^{2}$, anti-Streptococcus pneumoniae conjugada $^{3}$, anti-pertussis acelular ${ }^{4}$, anti-hepatitis $B$ en recién nacidos y lactantes ${ }^{5}$, anti-hepatitis $A^{6}$, anti-influenza en niños bajo 2 años de edad ${ }^{7}$.

- Detectar aumentos o disminuciones de efectos adversos conocidos: por ejemplo, documentar la desaparición de polio asociada a vacuna al cambiar de vacuna viva atenuada de uso oral a vacuna inacti- 
vada y la reducción de los efectos adversos asociados a vacuna anti-pertussis al cambiar de vacuna de células completas a vacuna acelular8.

- Detectar eventos de rara ocurrencia como: invaginación intestinal y vacuna anti-rotavirus ${ }^{9,10}$, enfermedad viscerotrópica y vacuna anti-fiebre amarilla, miocarditis y eventos isquémicos cardíacos y vacuna anti-variólica.

- Monitorizar seguridad por lotes: se realiza una revisión semanal de los SAEs por oficiales médicos de la FDA y cualquier agrupación de eventos similares se investiga ( sin embargo, desde el inicio del VAERS no se han retirado lotes de vacunas por razones de seguridad).

- Identificar potenciales factores de riesgo para EAAV: enfermedad viscerotrópica y vacuna antifiebre amarilla relacionada a edad avanzada, síncope post vacuna relacionado a edad (adolescentes y adultos jóvenes).

- Determinar la existencia o no de relación causal entre ciertos efectos adversos y ciertas vacunas: por ejemplo, se ha podido establecer que existe relación entre edema con cianosis o púrpura en el sitio de inyección y vacuna anti-Hib, y descartar la relación de eventos como muerte súbita con vacuna DTP, esclerosis múltiple y otras enfermedades desmielinizantes con vacuna anti-hepatitis $\mathrm{B}$, enfermedad de Crohn y autismo con vacuna tresvírica, autismo con timerosal en las vacunas ${ }^{11}$.

Limitaciones del VAERS. El sistema no está exento de limitaciones, las que deben tenerse en cuenta para un correcto uso y análisis de la información que entre$\mathrm{ga}^{12}$.

- Subnotificación: siendo un sistema pasivo, que recibe reportes voluntarios, la eficiencia de reportes es muy variable. Se ha estimado que la eficiencia de reporte puede variar entre $72 \%$ en los casos de polio asociada a vacuna oral, $50 \%$ en invaginación asociada a vacuna anti-rotavirus y $1 \%$ en exantemas post vacuna tresvírica. Generalmente, los reportes son más frecuentes en eventos graves, vacunas nuevas y están muy influenciados por la publicidad.

- Definiciones de caso: inicialmente el sistema funcionaba sin ellas, el primer esfuerzo en homogenizar los diagnósticos fue la definición operativa de los episodios de hipotonía-hiporespuesta relacionados a vacuna anti-pertussis. Actualmente, el sistema cuenta con definiciones de caso, pero aún existen limitaciones en el sistema de códigos utilizado. Una iniciativa, conocida como Brigthon Collaboration, trabaja en la estandarización de definiciones y categorización de la certeza diagnóstica.
- Cálculo de tasas de incidencia: considerando que no se dispone del reporte de todos los efectos adversos ocurridos, la falta de confirmación de los eventos y de causalidad y que no se conoce el denominador con certeza, los datos del VAERS no permiten calcular la incidencia de EAAV.

- Alternativamente a calcular tasas de incidencia de efectos adversos, se han utilizado las "tasas de reporte": EA reportados/ dosis distribuidas. Sin embargo, la información de dosis distribuidas no es igual al número de personas vacunadas, no entrega información por grupos etarios y no da información de distribución de lotes.

- Cálculo de riesgo relativo (RR): el RR es un radio de tasas de incidencia, por lo anteriormente expuesto, tampoco es posible calcular RR de ocurrencia de EAAV.

- Test de significancia e intervalos de confianza: por los múltiples sesgos del VAERS, no deben aplicarse rutinariamente pruebas de significancia estadística ni intervalos de confianza, o hacerlos con máxima cautela.

- Se han desarrollado métodos alternativos, conocidos como "data mining techniques", una revisión de éstos va más allá de los alcances de este documento.

\section{Errores frecuentes en el uso de la}

\section{información otorgada por el VAERS}

- Calcular tasas de incidencia de evento adverso, riesgo relativo, aplicar prueba de significancia estadística e intervalos de confianza.

- Considerar los reportes del VAERS como sinónimo de casos identificados de muertes e injurias causadas por vacunas: hay que considerar que la mayoría de los reportes corresponden a una relación puramente temporal con la vacuna y no necesariamente a una relación causal, la evaluación de causalidad requiere de mayor investigación.

- Atribuir ciertos eventos adversos como relacionados a ciertos grupos etarios: por ejemplo esclerosis múltiple en trabajadores de salud con vacuna antihepatitis $\mathrm{B}$, y muerte súbita, con vacunación en el primer semestre de vida. En realidad estos eventos adversos aparecen en las mismas edades en que la vacunación está indicada.

Los sistemas de VEAV dan señales que deben dar origen a investigaciones clínicas o epidemiológicas, idealmente estudios controlados, para establecer la posible relación de causalidad con la vacuna. 


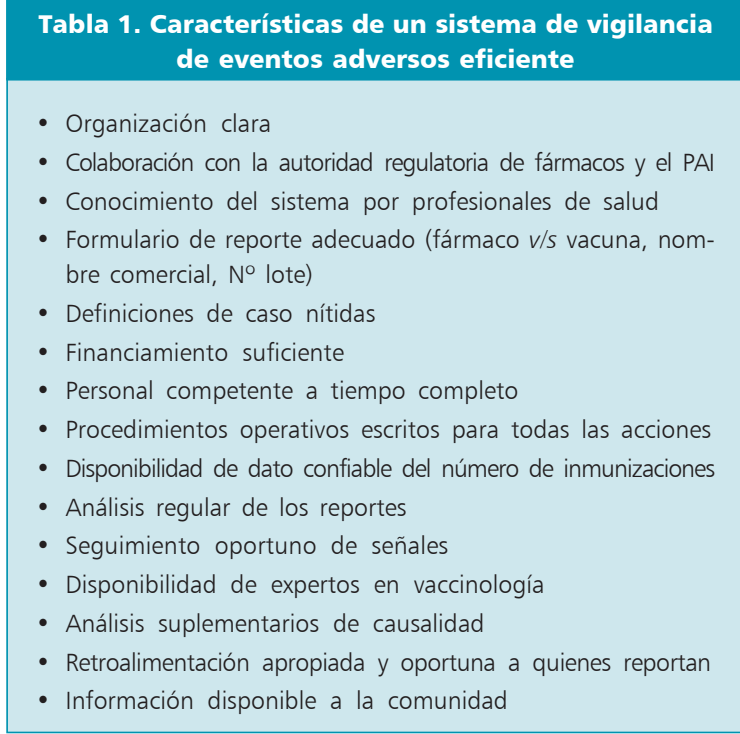

\section{Características de un sistema de VEAV eficiente}

Un estudio europeo investigó las características de los sistemas de VEAV en los países europeos, enunciando las características que debiera tener un sistema de VEAV eficiente (Tabla 1$)^{13}$.

Los principales problemas detectados en los sistemas de VEAV europeos fueron: falta de personal a tiempo completo, falta de financiamiento, falta de definiciones de caso, falta de denominadores, falta de adecuado análisis y retroalimentación inapropiada o inexistente.

En conclusión, se presenta una panorámica de los sistemas de VEAV en el mundo y en Chile, con sus aportes y limitaciones. Se espera que con estos antecedentes y un mejor análisis del sistema chileno, la comunidad médica y las autoridades sanitarias puedan analizar la pertinencia de realizar cambios que permitan optimizar este sistema, con miras a asegurar a la población chilena los mejores estándares de seguridad de las vacunas que se le administran.

\section{Resumen}

El propósito de los sistemas de vigilancia de eventos adversos a vacunas es monitorizar aquellos temporalmente relacionados con las vacunaciones, para evaluar su causalidad y para describir eventos acaecidos luego de la introducción de nuevas vacunas. El fin último de estos sistemas es proporcionar a la población el mejor estándar de seguridad relativo a vacunas. El sistema de vigilancia puede ser pasivo (reportes espontáneos) o activo (seguimiento activo de los vacunados); la mayoría en ejercicio es pasiva. Este artículo presenta un resumen abreviado de los sistemas de vigilancia de eventos adversos a vacunas más conocidos, incluyendo los sistema empleados en los Estados Unidos de América (Vaccine Adverse Events Report System-VAERS), Canadá y Europa, como así también una referencia al sistema chileno, destacando las principales ventajas y limitaciones en cada caso.

\section{Referencias}

1.- Iskander J, Miller E R, Chen R T. The role of the Vaccine Adverse Event Reporting System (VAERS) in monitoring vaccine safety. Pediatr Ann 2004; 33: 599-606.

2.- Wise R P, Salive M E, Braun M M, Mootrey G T, Seward J F, Rider L G, et al. Postlicensure safety surveillance for varicella vaccine. JAMA 2000; 284: 1271-9.

3.- Wise R P, Iskander J, Pratt R D, Campbell S, Ball R, Pless R P, et al. Poslicensure safety surveillance for 7-valent pneumococcal conjugate vaccine. JAMA 2004; 292: 1702-10.

4.- Braun M M, Mootrey G T, Salive M E, Chen R T, Ellenberg S S. Infant immunization with acellular pertussis vaccines in the United States: assessment of the first two years' data from the Vaccine Adverse Event Reporting System (VAERS). Pediatrics 2000; 106: E51.

5.- Niu M T, Davis D M, Ellenberg S.
Recombinant hepatitis B vaccination of neonates and infants: emerging safety data from the Vaccine Adverse Event Reporting System. Pediatr Infect Dis J 1996; 15: 771-6.

6.- Niu M T, Salive M, Krueger C, Ellenberg S S. Two-year review of hepatitis A vaccine safety: data from the Vaccine Adverse Event Reporting System (VAERS). Clin Infect Dis 1998; 26: 1475-6.

7.- McMahon A W, Iskander J, Haber P, Chang S, Woo E J, Braun M M, et al. Adverse events after inactivated influenza vaccination among children less than 2 years of age: analysis of reports from the Vaccine Adverse Event Reporting System, 19902003. Pediatrics 2005; 115: 453-60.

8.- Geier D, Geier M. An evaluation of serious neurological disorders following immunization: a comparison of whole-cell pertussis and acellular pertussis vaccines. Brain \& Development 2004; 26: 296-300.
9.- CDC. Intussusception among recipientes of rotavirus vaccines, United States, 19981999. MMWR Morbid Mortal Wkly Rep 1990; 48: 577-81.

10.- Davis R, Kolczak M, Lewis E, Nordin J, Goodman M, Shay D, et al. Active surveillance of vaccine safety. A system to detect early signs of adverse events. Epidemiol 2005; 16: 336-41.

11.- Autret-Leca E, Jonville-Bera A P, Beau-Salinas F. Vaccines pharmacovigilance. Rev Prat 2004; 54: 526-31.

12.- Varricchio F, Iskander J, Destefano F, Ball R, Pless R, Braun M M, et al. Understanding vaccine safety information from the Vaccine Adverse Event Reporting System. Pediatr Infect Dis J 2004: 23: 28794.

13.- Lankinen K, Pastila S, Kilpi T, Nohynek H, Mäkelä H, Olin P. Vaccinovigilance in Europe-need for timelines, standarization and resources. Bull World Health Organ. 2004; 82: 828-35. 